\title{
Fekete-Szegö Problems for Certain Class of Analytic Functions Associated with Quasi-Subordination
}

\author{
Pravati Sahoo
}

ABSTRACT: In this paper, we determine the coefficient estimates and the Fekete-Szegö inequalities for $\mathcal{M}_{q}^{\alpha}(\gamma, \lambda, \phi)$, the class of analytic and univalent functions associated with quasi-subordination. 